\title{
NON-SINGULAR REFERENCE: SOME PRELIMINARIES ${ }^{1}$
}

\section{F. JEFFRY PELLETIER}

One of the goals of a certain brand of philosopher has been to give an account of language and linguistic phenomena by means of showing how sentences are to be translated into a "logically perspicuous notation" (or an "ideal language" - to use passé terminology). The usual reason given by such philosophers for this activity is that such a notational system will somehow illustrate the "logical form" of these sentences. There are many candidates for this notational system: (almost) ordinary first-order predicate logic (see Quine [1960]), higher-order predicate logic (see Parsons [1968, 1970]), intensional logic (see Montague [1969, 1970a, 1970b, 1971]), and transformational grammar (see Harman [1971]), to mention some of the more popular ones. I do not propose to discuss the general question of the correctness of this approach to the philosophy of language, nor do I wish to adjudicate among the notational systems mentioned here. Rather, I want to focus on one problem which must be faced by all such systems - a problem that must be discussed before one decides upon a notational system and tries to demontrate that it in fact can account for all linguistic phenomena. The general problem is to determine what we shall allow as linguistic data; in this paper I shall restrict my attention to this general problem as it appears when we try to account for certain words with non-singular reference, in particular, the words that are classified by the count/ mass and sortal/non-sortal distinctions.

Nouns are normally divided into two classes: proper and common. Proper nouns themselves fall into two classes: those in one very rarely occur with a determiner, and those in the other usually with 'the' (Connecticut is a state, The Connecticut is a river). ${ }^{2}$ In the case of common nouns, there is general recognition that there are two quite distinct classes - at least "quite distinct" 
for the paradigms. The syntactical behavior of words like 'water', 'mud', and 'oatmeal' is quite different from the behavior of words like 'man', 'statue', and 'eye' [I shall call the former 'mass' and the latter 'count', in keeping with accepted usage]. For elementary purposes, textbooks (e.g. Gleason [1965]) often give criteria like the following: Count nouns are so-called because they can occur with numerals and can be used as either singular or plural. They admit of ' $a$ ' and 'every' with the singular, and 'few' with the plural. Mass nouns do not exhibit the singular-plural distinctions; when used as subjects, they take singular verbs. The determiners used with mass nouns, however, are more like those used with plural count nouns than those used with singulars. This may be brought out by a tabulation of grammatically comparable constructions (from Gleason [1965], p.135):

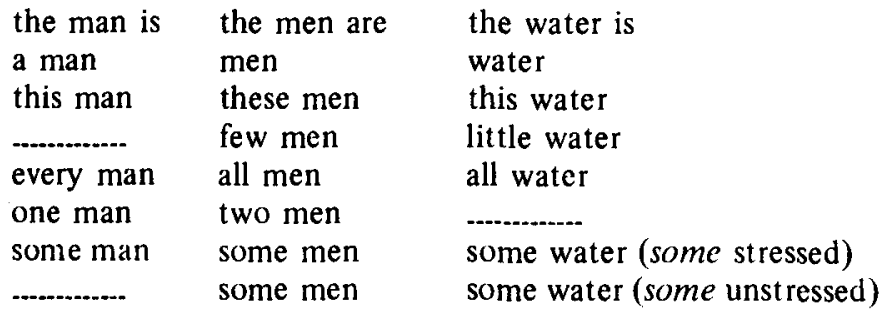

Unfortunately, this simple and elegant explanation of the syntactical difference between count and mass nouns will not suffice. First, it is claimed that count nouns, but not mass nouns, admit of numerals (and along with this is some related claim for pluralization). This at least needs some kind of qualification, for consider the (supposed) mass noun 'oatmeal': given an appropriate setting (such as a customer in a diner to his waitress), it is clear that such questions as 'How many oatmeals are in your kitchen?' have as a perfectly clear answer 'Three oatmeals', thus violating the two related criteria of mass nouns accepting neither pluralization nor numeral prefixes. Of course one might always retort "You've either changed the sense of 'oatmeal' or you have deleted from the surface structure some such phrase as 'kinds of' or 'bowls of, etc." True, I suppose I have, but such a claim makes clear that either (1) surface structure is not what the criteria are talking about or (2) we need to distinguish not between mass and count nouns but between mass and count senses of nouns. More about this will appear below, but for now it is well to cast a wary eye on such simplistic claims as we started with. 
The next criterion is pluralization: that mass nouns do not exhibit it. Even ignoring such difficulties as noted above, there are mass nouns that without change of sense admit of apparent (syntactical) pluralization: e.g.; 'beans' and 'potatoes' ('Pass the (mashed) potatoes', etc.) That is, it is the syntactically plural form that is used in the mass sense. Another rather straightforward attempt might be: mass nouns but not count nouns admit of the prefixes 'much' and 'amount of'. But as we shall see below, these expressions can be used with apparent count nouns.

There still remains more to the story of the count/mass distinction, but let's leave off for now and turn to a distinction given to use by traditional wisdom: sortal vs. non-sortal terms. Frege ([1884], p.66) explains for us:

The concept "letters in the word 'three" " isolates the ' $t$ ' from the ' $h$ ', and ' $h$ ' from the ' $r$ ', and so on. The concept "syllables in the word "three" " picks out the word as a whole, and as indivisible in the sense that no part of it falls any longer under the same concept. Not all concepts possess this quality. We can, for example, divide up something falling under the concept "red" into parts in a variety of ways, without the parts ceasing to fall under the concept "red". To a concept of this kind no finite number will belong. The proposition asserting that units are isolated and indivisible can, accordingly, be formulated as follows:

Only a concept which isolates what falls under it in a definite manner, and which does not permit any arbitrary division of it into parts, can be a unit relative to a finite number.

The distinction is supposed to divide predicates that "provide a criterion for counting" from predicates that do not provide such a criterion. In a space appropriate to the sortal ' $S$ ', we can count how many S's there are in that space; but in a space appropriate to a non-sortal ' $M$ ' we cannot straightforwardly ask how many M's there are. Thus we can ask how many men are in a room, but not how many waters (without changing the sense of 'water'). Nonsortal terms are collective - if ' $M$ ' is a non-sortal term, then ' $M$ ' is true of any sum of things of which ' $M$ ' is true - and divisive ' $M$ ' is true of any part of a thing of which ' $M$ ' is true (down to a certain lower limit, the setting of which is generally an empirical matter). 
This distinction is only clear in its broadest outline, and not much at all in the details, in spite of the fact that so much of the recent philosophical literature presupposes it. Nonetheless, we are in a position to compare, generally, the grammatical distinction, i.e., the count/mass distinction, with the philosophical one, i.e., the sortal/non-sortal distinction. That there are two distinctions here is, I think, insufficiently recognized; Wallace [1964] p.70 runs the two together, Gleason [1965] pp. 135-137 tries to show that count nouns do what we admit only sortals do, Quine [1960] uses the criteria for sortals and calls them count, and Moravcsik [1970], while recognizing the two ways to make these distinctions, uses the sortal/non-sortal distinction and thinks that he has adequately characterized the count/mass distinction.

It strikes me that there are five important differences between the two distinctions. First, the grammatical distinction applies only to nouns whereas the philosophical distinction is usually asserted to apply to all monadic predicates. For example, the grammatical distinction does not treat 'red' or 'spherical' at all, but the philosophical distinction is sometimes held to classify 'red' as a non-sortal and 'spherical' as a sortal. Second, the grammatical distinction applies only to simple nouns, whereas the philosophical distinction applies to complex terms. E.g., it makes 'white man' a sortal and 'dirty water' a non-sortal. Third, certain count nouns are classified as non-sortals, e.g., 'thing', 'object', 'entity'. Fourth, the grammatical distinction will make abstract nouns such as 'speed' and 'knowledge' mass nouns, and make 'plot' and 'virtue' (in one sense) count. The philosophical distinction is vague at this point; some philosophers do, but others do not, want the distinction to apply to such terms at all. And fifth, measures on mass nouns (e.g., 'lump of coal', 'gallon of gasoline') raise special problems. Some of these are divisive (e.g., 'lump of coal' or 'amount of dirt') and would probably be classified as non-sortal (although Wallace [1964] calls 'lump of coal' sortal and 'amount of dirt' non-sortal). But others are more problematic: for instance, 'blade of grass' is not obviously divisive or collective. There are certain affinities between these "measured mass nouns" and "counted count nouns" (e.g., "busload of teams of basketball players'), but I shall not pursue them here. ${ }^{3}$ In fact, I shall completely ignore these occurrences of mass terms in what follows. In any case, all of these "measured mass nouns", even 'amount of dirt', if treated at all, would be classified as count by the grammatical distinction.

The difference between the distinctions is a matter of focus. 
The grammatical distinction is supposed to describe the syntax of our language - it tries, without theory, to show us how to tell the one kind of word from the other. It is supposed to be a starting point for a theory - that is, it is supposed merely to describe some phenomenon that any general account (i.e., theory) of language must face up to. For this reason, in order to succeed, the distinction must not appeal to any theory but only to surface structure and other pre-theoretic information. (In fact, the distinction cannot appeal to the theory in which it is being used without being circular. $)^{4}$ We've seen above that for the criteria given to work, we must (1) have recourse to structures other than surface structure, or (2) be able to distinguish senses of words before we apply the criteria. Now, (1) is clearly theory-laden, and so this preliminary distinction can have no recourse to such structures; but (2) seems different - one would like to think that the ability to distinguish different senses of the same word is pre-theoretic. We should, therefore, be able to use at least some of the criteria given to make the desired mass/count distinction (e.g., we should be able to give as a criterion: When $X$ is used in a mass sense, phrases like 'an amount of $X$ ' and 'much $X$ ' are not anomalous; when $X$ is used in a count sense, phrases like 'an $X$ ' and 'four X's' are not anomalous; but the reverse is not true).

But is (1) so clearly theory-laden? The fact that speakers can discriminate between senses of sentences might be taken as evidence supporting a deep structure analysis. ${ }^{5}$ One consideration which can be brought out as a reason for rejecting this support for (1) is: speakers can indeed tell that sentences are ambiguous, but sometimes they can also pinpoint where the ambiguity comes from. In the case given here, they will point to the word 'oatmeal'. It is no good to say that the informants might not be able to say what the ambiguity is or to what it is due in any theoretically interesting way. Such claims find their home in the analysis of sentences like 'Two soldiers shot two students'; in such sentences informants may well be unable to give an accounting of the ambiguity. But at least they will be able to discern that it is not due to any lexical ambiguity. Conversely, when they can attribute the ambiguity to a lexical item, as in the present case, that cuts in favor of (2), and against (1).

1 think that reflection on the example of above, 'How many oatmeals are in your kitchen?' provides convincing evidence that every word which would normally be called a mass noun can be given a perfectly clear count sense. This sense might be the same 
as that of 'kind of oatmeal', or 'bowl of oatmeal', etc., or it may be different. There are many nouns which have mass/count senses related in other ways. 'I like chickens' versus 'I like chicken', 'Pass the five potatoes' versus 'Pass the (mashed) potatoes' show the distinction occurring between a naturally-constituted object (i.e., structured according to biological, geological, or cultural norms, ${ }^{6}$ etc.) and the matter of which it is made.

Can all words that one is tempted to call count nouns be given a mass sense? A "thought experiment" like the following might be described in order to persuade one that it is possible to do so. Let's agree that a mass or count sense of a word exists if one can describe a circumstance or set of circumstances in which that word with the requisite sense can (or would) be normally employed. Consider a machine, the "universal grinder".? This machine is rather like a meat grinder in that one introduces something into one end, the grinder chops and grinds it up into a homogeneous mass and spews it onto the floor from its other end. The difference between the universal grinder and a meat grinder is that the universal grinder's machinery allows it to chop up any object no matter how large, no matter how small, no matter how soft, no matter how hard. Now if we put into one end of a meat grinder a steak, and ask what is on the floor at the other end, the answer is 'There is steak all over the floor' (wherein 'steak' has a mass sense). It may be true that we have a special term for the mass sense of 'steak', such as 'ground sirloin', but in general this will not be the case. And in any case, it is only relevant to note that the sense of 'steak' in the answer given above is mass, and the answer is normal. The reader has doubtless guessed by now the purpose of our universal grinder: Take an object corresponding to any (apparent) count noun he wishes (e.g., 'man'), put the object in one end of the grinder and ask what is on the floor (answer: 'There is man all over the floor'). Perhaps there are other answers to this question, such as 'There are pieces of a man all over the floor', but this is irrelevant to the test. All that needs be the case is for one of the possible normal answers to use the mass sense of our "normal" count noun, and this has been supplied. It is apparent that this test can be employed at will, always giving us a mass sense of count nouns having physical objects as their extension.

There is still some question about nouns which do not have physical objects in their extension - ungrindable things like unicorns (ungrindable because there are none of them to grind): 
are they the only true count nouns? The answer is no: it is not necessary that the object actually be grindable, but only that a normal sentence use the word in a mass sense. The sentence 'If there were any unicorns and if we were to put one into the grinder, there would be unicorn all over the floor' uses 'unicorn' in the required sense. A harder example is 'number', but perhaps one would be satisfied with the sentence 'If numbers were physical objects, and if we were to put one into the grinder, there would be number all over the floor's At any rate, there can be made a prima facie case that nothing is immune from the grinder treatment. So there is at least a prima facie reason to believe that every noun must have (perhaps hidden) both a count and a mass sense. Reasoning similar to this is used by Gleason [1965], pp.136-137:

Are there limitations to this shifting [between count and mass senses]? At first there seems to be.... But it is soon found that many of the ones with both uses are very much more frequent in one than in the other. The less frequent use occurs only in rather unusual circumstances. Water as a mass noun is common and widespread; as a count noun is nearly restricted to waiters. Even if the restaurant usage had not been observed, the pattern would remain and this use might arise at any time. Perhaps some of the other words would also show both uses if sufficiently unusual situations were conceived. This seems to be the case. For example, book and shelf are both fairly typical count nouns. With the present vogue for speaking -- animal stories, we can imagine one featuring a mother termite concerned over her child: Johnny is very choosey about his food. He will eat book, but he won't touch shelf. This is farfetched, of course. But it does suggest that every noun, given the right context can occur in either type of usage, count or mass.

There are two kinds of objections one can make to this. First, we might object to this use of counterfactual: if the counterfactual's antecedent is contradictory, can we always be assured of grammaticality? In cases like 'If 4 were the smallest even prime number, then 2 would not be' we have clearly true (and hence grammatical) sentences. But with sentences like 'If all of mathematics were entirely different than it is, then 4 would be the smallest even prime number' we are quite at a loss. Does the 'If numbers were physical objects...' belong to the first or the second 
class? Furthermore, what about such words as 'individual' or 'thing'? It seems that if an individual or a thing is put into the grinder, then (in the absence of any other information about what the individual or thing is) the only natural answer is that there is stuff all over the floor. Such problems restrict the extent of Gleason's claim. We might also try to deny the applicability of the grinder in particular and "thought experiments" in general by calling into question the underlying assumption that a sense of a word exists if one can describe circumstances wherein that sense would normally be employed. The matter cannot easily be put aside by simply saying, "We are not interested in how language might be if the world were different; we are interested in the structure of English now." Or in Grandy's language (see fn.1), if the world were different, and water, sand, etc., came in large, relatively stable and permanent chunks, we would no doubt speak of sands, waters, etc. "If the world were different, our syntax (as well as our beliefs and semantics) would probably change as well. This should not be a surprising discovery..." But this is not exactly what is at issue here. If one looks at the discussion of a few pages ago, he will notice a series of sentences in which the phrase 'universal grinder' occurs. Also there will be various occurrences of 'man' that one has to understand in a mass sense. Furthermore, all of it is written in present-day English - thus this phenomenon arises within language, and is not simply a peculiarity of the relation between language and the world. But this is giving too much. We would not say that 'Ugga Bugga bo' is grammatical English, even though it makes sense when considered as part of the series of sentences: "When I say 'Ugga Bugga bo' I mean that grass is green. Ugga Bugga bo". Perhaps the reason this is an unacceptable example is that, intuitively speaking, we would call this a code, and furthermore because sentences of this sort are not uniformly generated. But this is not all, and I do not know the rest. At any rate, every obvious criterion I can think of either rules out suppositions from being English or else admits the "grinder" as generating present-day English uses of ordinary count terms in a mass sense.

In contrast to this descriptive purpose stands the purpose of the philosophical distinction, which is not intended to give a syntactical characterization but rather something we might want to call a semantical one. The criteria usually proffered (as by Frege above) tell us that we should look to the reference of the term in question: if it "provides a criterion for counting" or if it is not 
divisive or if it is not collective then it is sortal: otherwise it is non-sortal. (The results obtained above should be kept in mind. Namely, it seems that many predicates - almost all nouns - have both a sortal and non-sortal sense.)

Using the semantic criteria to judge whether a word (or word sense) is mass or count will lead to impossible difficulties. Any criterion which concerns itself with the nature of the reference of a word will find no interesting differences between 'thing' and 'water'. To hold (as Quine [1960] and Moravcsik [1970] do) that such criteria are sufficient to make this distinction, will be to hold that a syntactical theory cannot make use of the distinction (even if it were allowed that semantics can be settled before developing the related syntax). For any syntactical theory will group 'thing' with 'man' and its like, and it will separate this group from the group containing 'water' and its like. The reason for this grouping is, of course, that 'thing' admits the same prefixes, figures in the same kinds of transformations, etc., as does 'man' - and these are radically different from the ones 'water', etc., admit of. So a syntactical theory could not use the Quine-Moravcsik distinction because it does not group in this way, and the point of making the distinction at all will be lost (except of course for its philosophical interest, but this is then the sortal/non-sortal distinction and ought to be called such. The philosophical distinction would be of little interest for anyone constructing a syntactical theory). Both Quine and Moravcsik would find it difficult to give an account of a sentence containing 'thing'. According to the semantic criteria given by Quine, 'thing' is mass, so a sentence containing 'things' (plural) must be either ill-formed or else elliptical for a more complex sentence. (As e.g., when we use a sentence containing 'waters' - as 'There are two waters in this room', this is elliptical for something like 'kinds of water' or 'bodies of water' - as in 'There are two kinds of [or 'bodies of', etc.] water in this room'). One should note that the apparent count use gets replaced by an individuator term (measure?) plus mass use of the term. But clearly the sentence 'There are two things in this room' is not ill-formed, so it must be elliptical for 'There are two kinds of thing in this room' (where 'thing' has its "true" mass sense). However, the first sentence can be true when the second is false, as when we have two distinct things falling under all the same kinds (as perhaps if there are two men in the room, or two atoms in the room, or whatever one's metaphysical biases dictate). The problem is that 'thing' is a count noun, and any syntactical 
account must recognize this fact. Giving semantic criteria along the lines that Moravcsik and Quine have suggested will never make 'thing' come out count.

Part of the problem here seems to be that we want to cling to the grammatical distinction because it stands some chance of being clearly made (bearing in mind the above discussion about words vs. senses of words). But on the other hand it does not make quite the distinction one wants for philosophical purposes. Thus the goal should be now to show how to "generate" the sortal/non-sortal distinction from the mass/count distinction; i.e., to show how to overcome the five differences noted above. (From now on l will use 'term' or 'noun', etc., but in keeping with the discussion, I mean senses of the terms or nouns.) We can get rid of the first and fifth differences by merely expanding the grammatical distinction to include complex terms and from this strike out measures on mass terms (like 'lump of coal', 'gallon of water') -- the latter for separate treatment. The second dispute, that the philosophical distinction treats even non-substantial phrases (like adjectives), I think should be resolved in favor of the grammatical distinction i.e., the philosophical distinction should not attempt to classify these terms as either sortal or non-sortal. To understand why, we should look at the accounts given by those who think that, for example, adjectives should be so classified. In the Frege quote there is a certain problem surrounding his statement "we can divide something falling under the concept 'red' in a variety of ways..." What is it that we are diving up? Surely it is not the red, for consider the red book on my desk, and contrast it with the coffee which is also on my desk. To show that 'coffee' is a non-sortal term, the coffee on my desk must be divided to see if each of these parts of my original coffee are coffee (they are). However, the red on my desk is divided by dividing the red book. These parts of $m y$ book must be examined to determine if they are red (they are, if I started out with an entirely red book). The point is that we are to divide up the $X$, and see if the parts are still $X \ldots$ we are not supposed to divide up $Y$ and see if those parts are still $X$. There is a sense in which we can divide up the red, but this is a sortal sense of noun ("How many reds are there on the desk?" "Three: scarlet, crimson, and brick"). And even if we ignore this problem, we still cannot give a clear sense to sortal or non-sortal senses of adjectives. Words such as 'heavy' and 'light' would seem to be examples of non-sortal terms, since they do not "divide their reference into discrete objects". But also they do not 
seem to pass the divisibility test (not all parts of things that are heavy are themselves heavy) nor the collectivity test (not all sums of things are are light are themselves light). ${ }^{9}$ I think this should justify us giving up the idea that adjectives and other nonsubstantival terms can have sortal or non-sortal senses.

The fourth point of dispute, terms with non-physical objects in their extension, seems to me to be best settled by not having the philosophical distinction applied to them at all --i.e., strike them from the (revised) grammatical distinction which we have when we are generating the sortal/non-sortal distinction. The reason for this is that such terms have no "appropriate space" within which to judge whether it is or is not possible to count how many there are. Consider the most likely candidates for being sortal: words for geometrical figures (in the abstract sense) like 'triangle', 'square', etc. It is clear that the main sense of these sorts of words are count, since they admit numerical prefixes, can be used with ' $a$ ' and 'every', etc. However, one of the purposes of the 'sortal' designation is, as Frege notes, to be able to apply a number to it in a definite manner, and not to permit any arbitrary division of it into parts. Yet, since an abstract geometrical figure needs no physical bounds, it follows that there are an indefinite number of triangles within each triangle. ${ }^{10}$ And this violates the intent of the sortal designation. Yet it is also not right to call them non-sortal, for it is not true that there is a space appropriate to 'triangle' such that one cannot count how many there are in that space (as there was a space appropriate to 'water' but one could not count how many there were in that space).

Only the third difference, non-sortal count terms, remains to be settled. Some examples of these are 'thing', 'white thing', and 'physical object'. According to traditional wisdom, some phrases containing these are sortal, some not. Predicates like 'thing that is wise' or 'physical object that is thinking of Vienna' are sortal, since only a person could be wise or think of Vienna (and 'person' is a sortal predicate). On the other hand the simple phrases 'physical object', 'white thing', etc., are taken to be non-sortal since they pick out no definite thing or things - do not provide a criterion for counting, are divisive, etc. - to which a property can be applied. Many people (e.g., Geach [1962], p.148) brand sentences with such phrases as 'meaningless', because they do not refer to a particular thing or group of things. But surely the "meaningless phrases" can, in the following sentences, be interpreted in at least one way that makes sense (and they might even 
be true): 'Every physical object is extended', 'All white objects are white'. The problem is to understand just what the interpretation shall be.

I suggest the following: In the sentences where 'thing' (or a phrase containing 'thing') is being used as a proxy for some other term (as in 'thing that is wise') if 'thing' is replaced by what it is going proxy for, reflection will show that the replacement will always be by a sortal. ('Thing that is wet' cannot be replaced by 'water', rather 'stuff that is wet' is what 'water' can replace.) In the remaining (non-proxy) cases we should take the sentence in the question to be a quantification over (normal) sortals. E.g., 'All white things are white' is to be construed as 'For all sortals $S$, and any $x$, if $x$ is a white $S$, then $x$ is white', and so on. This explains both why 'thing' is a count noun (since it either "stands for" or indicates quantification over sortals) and also why it is not itself a sortal noun." Thus in any proposed "logically perspicuous notation" there should be no word 'thing' or phrase 'physical object' since they should all be replaced by a sortal or by a quantifier phrase of some sort.

A similar phenomenon appears to happen on the mass noun side also. Sentences like 'Water is wet', 'Mercury is dry', etc., are perfectly normal (from both the grammatical and philosophical point of view) and yet 'All stuff is fluid in nature' seems to pass only the tests for grammaticality, but not those for straightforward intelligibility, since no definite stuff has been picked out for us to say something about or predicate anything of. Words like 'stuff seem to have the two uses corresponding to those we distinguished for words like 'thing' - first, as a proxy for a normal mass noun, and second, as an indication of quantification over (normal) stuffs. The above sentence, for example, is to be construed as 'For any (normal) stuff $M, M$ is fluid in nature'. For simplicity let's agree to call normal sortal predicates "sortals", normal mass nouns "mass nouns", 'thing' and its relatives "secondary sortals" and "super sortals" corresponding to whether it "goes proxy" for a sortal or it is construed as quantification over sortals, and "stuff' and its relatives "secondary mass nouns" and "super mass nouns" for the corresponding two cases for mass nouns. Again, no "logically perspicuous notation" should contain any of these sorts of words, but rather will have replace them by a normal mass noun or by some kind of quantification.

Thus for monadic noun phrases that are not measure on mass nouns at least, we have a pretty clear grasp of what is a sortal 
according to traditional wisdom. They are what I call sortals and secondary sortals. Non-sortals according to traditional wisdom are what I call super-sortals, together with all our mass nouns (normal, secondary, and super). So we see that the philosophic distinction can, in a way, be "generated" from the grammatical one. For instance, to get the sortals from the counts, all that we have to do is to (a) include all noun phrases, (b) delete abstract noun phrases and measures on mass nouns, (c) distinguish "proxy" and "super" count nouns from the rest, and (d) reconstrue these two kinds of count nouns correctly (find the right word it goes "proxy" for, or find the proper quantifier phrase).

I think I have sufficiently shown that it is not easy to make these two distinctions. What $I$ have not here demonstrated is that these distinctions must be accounted for in any philosophically adequate theory of language. Perhaps it is easy enough to see that the mass/count distinction should be preserved in a canonical notation, since it looms so large and on the surface of English grammar. ${ }^{12}$ As for the sortal/non-sortal distinction: if one takes any of a number of closely related views prevalent in recent philosophical literature, one will believe that sortal terms are "the glasses through which we view the world", or the solution to the "paradox of confirmation", or how we "identify" and "reidentify" objects, or how we "pick out domains of discourse", or how we learn to think and talk, etc., etc. Any proponent of such a position who also views the construction of a "logically perspicuous notation" as an aid in understanding our ordinary language, must insist on the inclusion of this distinction in the artificial language if it is to be of any help in achieving his goal.

UNIVERSITY OF ALBERTA EDMONTON 7, ALBERTA

CANADA

\section{NOTES}

1 The penultimate version of this paper was read at the Pacific APA meeting. April, 1972. I want to thank the meeting's chairman, J.M.E. Moravcsik, and my commentator, Richard Grandy, for their comments. I have not addressed myself to all the points raised by Grandy, but only to a few of the more important ones. Various still earlier versions of this paper were read by David Lewis, Barbara Hall Partee, and John Perry. The paper is considerably better as a result. 
2

Contra Quine [1960] p.90.

3 The interested reader will find at least a wealth of examples bearing on this similarity in Celce and Schwarcz [1969].

4 Already, of course, we have some theory invoked with the notion of "surface structure". It seems to me, though, that it would be difficult to find a clear boundary between theoretic and non-theoretic; rather, descriptions of phenomena form a continuum from one to the other. But we can at least judge that "deep structure" invokes more theory than "surface structure", and for that reason the latter is preferable as a description of the data upon which our theory is to be built.

5 This objection was raised by Grandy (see footnote 1).

'An example of each: 'blade of grass', 'vein of gold', 'ream of paper'. Of course, in the examples given, the noun in question is clearly count without the help of an explicit "constitutional" term like "blade of, 'vein of or 'ream of'. So to speak, the noun in its count sense has "built into it" the "constitutional" feature of being a single chicken or single potato.

7 The "universal grinder" was suggested to me by a joke made by David Lewis in 1968.

8 This application of the "grinder" is also due to David Lewis.

9 This last criticism is from Moravcsik [1970], although he does not seem to believe its conclusion.

10 This point was brought to my attention by David Lewis in 1970.

11 This treatment is different from that given in Wallace [1964] pp.77-78, where he gives the "proxy" treatment essentially as I have done, and instead of considering the other cases higher level quantification he calls it "use as a philosophical substantive" which is "conceptual or theoretical in what it affirms" and is "not confirmed by its instances". All this seems to me to be false.

12 Although it should be noted that some people, e.g., Quine [1960], seem to think that the mass category is "archaic". Moravcsik's [1970] rejoinder to this is that it is only through mass concepts that quantative measurements, and thus scientific advancement, is possible.

\section{BIBLIOGRAPHY}

[1969] Celce, M. and Schwarcz, R.M. Counting, Collecting, Measuring, and Quantifying In Iinglish. (Santa Monica, Cal.: System Development Corporation document SP-3378).

[1884] Frege, Gottlob Die Grundlagen der Arithmetik. Translated by J.L. Austin as The Foundations of Arithemetic (Oxford: Basil Blackwell, 1950). References are to the translation.

[1962] Geach, Peter, T. Reference and Generality. (Ithaca, N.Y.: Cornell University Press). 
[1965] Gleason, H.A. Linguistics and Lnglish Grammar. (Toronto, Canada: Holt Reinhard Winston of Canada).

[1971] Harman, Gilbert "Deep Structure as Logical Form" Synthese.

[1972] Hintikka, K.J.J., Moravcsik, J.M.E. and Suppes, P. (eds.) Approaches to Natural Language (Amsterdam, Reidel).

[1969] Montague, Richard "On the Nature of Certain Philosophical Entities" The Monist.

[1970a] ____English as a Formal Language" Linguaggi Nella Societa e Nella Tecnica (Milan, Italy).

[1970b] "The Proper Treatment of Quantifiers in English" in Hintikka et al (eds.) \{1972\}.

[1971] _........... "Universal Grammar" Theoria.

[1970] Moravcsik, J.M.E. "The problem of the Semantics of Mass Terms in English" in Hintikka et al (eds.) [1972].

[1968] Parsons, Terrance "A Semantics for English" (unpublished paper).

[1970] "An Analy sis of Mass and Amount Terms" Ioundations of language (which is a part of Parsons [1968]).

[1960] Quine, W.V. Word and Object. (Cambridge, Mass.: M.I.T. Press).

[1964] Wallace, John "Philosophical Grammar" (unpublished Ph.D. dissertation, Stanford). 